$\Rightarrow$ TUMOUR MICROENVIRONMENT

\section{Macrophage manipulation}

molecular
mechanisms
behind the
metabolic
reprogram-
ming of
tumour-
associated
macrophages

Three studies have delved into the molecular mechanisms behind the metabolic reprogramming of tumour-associated macrophages (TAMs), which have consequences for tumour progression and therapeutic response and resistance.

TAMs are known to drive chemoresistance to gemcitabine (Gem) in pancreatic ductal adenocarcinoma (PDAC) and so Halbrook et al. directed their investigation into the metabolic crosstalk between macrophages and PDAC cells. Tumour-educated macrophages (TEMs) were generated by culturing mouse bone marrow-derived macrophages (BMDMs) in PDAC conditioned media (CM). Metabolomic profiling of the TEM CM showed release of pyrimidine nucleosides and nucleobases compared with PDAC $\mathrm{CM}$ alone. Furthermore, incubation of PDAC cells with TEM CM was sufficient to reduce the amount of many of these pyrimidines in line with directional metabolite exchange.

Given that Gem is a pyrimidine anti-nucleoside, the authors reasoned that release of pyrimidine species by TEMs might impede the therapeutic efficiency of Gem. To explore this idea, PDAC cells were treated with Gem in the presence of a high concentration of the nucleosides. This revealed that deoxycytidine

(dC) exclusively prevented

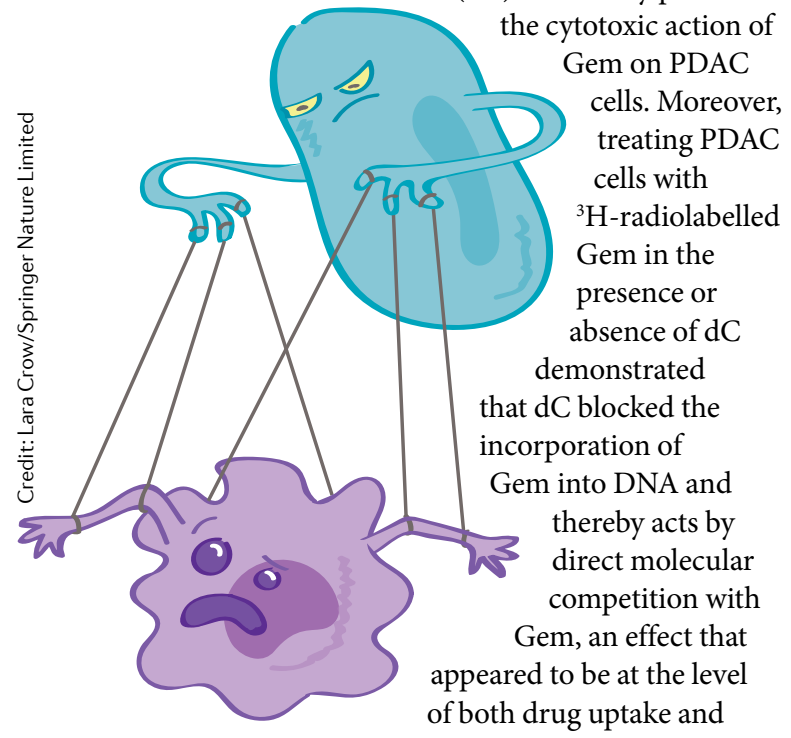

metabolism. Consistent with these observations, a cohort of patients with PDAC who had undergone surgery followed by adjuvant Gem treatment had significantly improved survival if they had a high macrophage burden compared with a low one.

Takenaka et al. also used tumour CM (TCM), but in this case from mouse GL261 and CT2A glioma cells and human glioblastoma samples, to study the effects of secreted metabolites on TAM function. As tumour-intrinsic aryl hydrocarbon receptor (AHR) signalling through the tryptophan-derived metabolite kynurenine had already been shown to influence glioblastoma progression, the authors chose to focus on this signalling pathway.

TCM increased the expression of Ahr and AHR target genes, such as Entpd1 (which encodes the ectonucleotidase CD39) in mouse macrophages. To explore this phenotype further, mice were generated in which the AHR gene is conditionally deleted in the myeloid cell lineage $\left(\mathrm{AHR}^{\mathrm{LysM}}\right)$, and then injected intracranially with GL261 cells. Under these conditions, tumour growth was decreased and associated with fewer TAMs and increased survival of the mice. Importantly, the authors noted that TAMs from AHR ${ }^{\mathrm{LysM}}$ mice had downregulated M2-like genes, typically associated with tumour progression and immunosuppression, and upregulated classically activated M1-like genes. Further experiments revealed that kynurenine controlled TAM polarization through an AHR transcriptional response involving KLF4 and SOCS2.

$\mathrm{AHR}^{\mathrm{LysM}}$ mice also exhibited reduced expression of CD39 in TAMs and higher numbers of $\mathrm{CD} 8^{+}$ tumour-infiltrating lymphocytes (TILs). In combination with the observation that mice with myeloid cell-specific deletion of Entpd1 also had more $\mathrm{CD} 8^{+}$TILs with low levels of genes related to $\mathrm{T}$ cell dysfunction, this suggested that AHR-driven
CD39 expression in TAMs might limit the $\mathrm{T}$ cell immune response through increased production of the immunosuppressive nucleotide adenosine in the TME.

Investigating how the metabolic environment of ovarian cancer might shape the tumour-promoting phenotype of TAMs, Goossens et al. intraperitoneally transplanted ovarian ID8 cancer cells into syngeneic mice. At a late time point when tumours were more established, TAMs isolated from ID8 tumour-bearing mice displayed enrichment of a set of genes related to cholesterol metabolism and efflux. Accordingly, these TAMs also had decreased membrane cholesterol content. Incubation of BMDMs with ID8 CM confirmed that tumour cells could directly promote the depletion of membrane cholesterol from macrophages and that the tumour-derived secreted factor driving this effect was the extracellular matrix molecule hyaluronic acid.

Macrophage activation is known to be altered by variations in membrane cholesterol content in response to pro-inflammatory stimuli. In agreement with this, ID8 CM reprogrammed BMDMs toward an interleukin-4 (IL-4)mediated tumour-promoting phenotype. Finally, to demonstrate that cholesterol efflux regulates the macrophage activation state in vivo, the researchers genetically deleted the $A B C$ cholesterol efflux transporters ABCA1 and ABCG1 in the myeloid cell lineage of mice. ID8 tumours established in these mice exhibited inhibited tumour growth while the IL-4-induced pro-tumour phenotype of TAMs was reversed.

Together, these studies offer new possible therapeutic targets to restore the antitumour potential of TAMs.

Anna Dart

ORIGINAL ARTICLES Goossens, P. et al. Membrane cholesterol efflux drives tumorassociated macrophage reprogramming and tumor progression. Cell Metab. https://doi.org/ 10.1016/j.cmet.2019.02.016 (2019) |Halbrook, C. J. et al. Macrophage-released pyrimidines inhibit gemcitabine therapy in pancreatic cancer. Cell Metab. https://doi.org/10.1016/j.cmet.2019. 02.001 (2019) | Takenaka, M. C. et al. Control of tumor-associated macrophages and T cells in glioblastoma via AHR and CD39. Nat. Neurosci. https://doi.org/10.1038/s41593-019-0370-y (2019) 